\title{
Supplementary Information: Approximate Bayesian Computation and simulation-based inference for complex stochastic epidemic models
}

Trevelyan J. McKinley, Ian Vernon, loannis Andrianakis, Nicky McCreesh, Jeremy E. Oakley, Rebecca N. Nsubuga, Michael Goldstein and Richard G. White

\section{S1. BISECTION METHOD FOR CHOOSING TOLERANCES AT EACH GENERATION OF ABC}

A1. Denote tolerances at generation $t$ as $\tau_{k}^{t}$ for $k=1, \ldots, K$.

A2. Set a value $p_{\tau} \in(0,1)$ denoting the target acceptance rate (e.g. the target proportion of particles in generation $t$ to retain in generation $t+1$ ).

A3. Set upper and lower bounds for $p_{\tau}$, denoted $p_{\tau}^{U}$ and $p_{\tau}^{L}$ respectively.

A4. For each output $k=1, \ldots, K$, set a lower bound for the proposed tolerances, $\tau_{k}^{L}$ as the $p_{\tau}$-percentile of the simulated metric distances at generation $t$.

A5. Set an upper bound for the proposed tolerances, $\tau_{k}^{U}=\tau_{k}^{t}$.

A6. Set an initial proposal for each tolerance as $\tau_{k}^{*}=\tau_{k}^{L}$.

A7. Calculate the proportion of generation $t$ particles that match according to $\tau_{1}^{*}, \ldots, \tau_{K}^{*}$ Denote this $p^{*}$.

A8. If $p_{\tau}^{L}<p^{*}<p_{\tau}^{U}$ then set $\tau_{k}^{t+1}=\tau_{k}^{*}$ for all $K$ outputs and stop.

A9. If $p^{*}<p_{\tau}^{L}$ or $p^{*}>p_{\tau}^{U}$, then set

$$
\tau_{k}^{L}=\tau_{k}^{*} \text { and } \tau_{k}^{*}=\left(\tau_{k}^{U}+\tau_{k}^{*}\right) / 2 \text { if } p^{*}<p_{\tau}^{L},
$$

or

$$
\tau_{k}^{U}=\tau_{k}^{*} \text { and } \tau_{k}^{*}=\left(\tau_{k}^{L}+\tau_{k}^{*}\right) / 2 \text { if } p^{*}>p_{\tau}^{U}
$$

and go to step A7.

Algorithm S1: Bisection method for generating tolerances at each generation 